\title{
Hemispheric asymmetries in subcortical visual and auditory relay structures in congenital deafness
}

\author{
L. Amaral, ${ }^{1,2,3}$ A. Ganho-Ávila, ${ }^{1,2,4}$ A. Osório, ${ }^{5}$ M. J. Soares,${ }^{1,2}$ D. He, ${ }^{6}$ Q. Chen, ${ }^{7}$ B. Z. Mahon, ${ }^{7,8,9}$ \\ O. F. Gonçalves, ${ }^{4,10,11}$ A. Sampaio, ${ }^{4}$ F. Fang, ${ }^{6,12,13}{\mathrm{Y} . ~ B i^{14}}^{14}$ and J. Almeida ${ }^{1,2,3}$ \\ ${ }^{1}$ Proaction Laboratory, Faculty of Psychology and Education Sciences, University of Coimbra, 3001-802 Coimbra, Portugal \\ ${ }^{2}$ Faculty of Psychology and Education Sciences, University of Coimbra, Coimbra, Portugal \\ ${ }^{3}$ CINEICC, Faculty of Psychology and Education Sciences, University of Coimbra, Coimbra, Portugal \\ ${ }^{4}$ Neuropsychophysiology Laboratory, Research Center in Psychology, School of Psychology, University of Minho, Minho, \\ Portugal \\ ${ }^{5}$ Social and Cognitive Neuroscience Laboratory and Developmental Disorders Program, Center for Health and Biological \\ Sciences, Mackenzie Presbyterian University, Sao Paulo, Brazil \\ ${ }^{6}$ Department of Psychology and Beijing Key Laboratory of Behavior and Mental Health, Peking University, Beijing, China \\ ${ }^{7}$ Department of Brain and Cognitive Sciences, University of Rochester, Rochester, NY, USA \\ ${ }^{8}$ Center for Visual Science, University of Rochester, Rochester, NY, USA \\ ${ }^{9}$ Department of Neurosurgery, University of Rochester, Rochester, NY, USA \\ ${ }^{10}$ Bouvé College of Health Sciences, Northeastern University, Boston, MA, USA \\ ${ }^{11}$ Department of Physical Medicine and Rehabilitation, Spaulding Rehabilitation Hospital, Harvard Medical School, Boston, MA, \\ USA \\ ${ }^{12}$ PKU-IDG/McGovern Institute for Brain Research, Peking University, Beijing, China \\ ${ }^{13}$ Peking-Tsinghua Center for Life Sciences, Peking University, Beijing, China \\ ${ }^{14}$ State Key Laboratory of Cognitive Neuroscience and Learning, Beijing Normal University, Beijing, China
}

Keywords: auditory cortex, neuroimaging, neuronal plasticity, ocular vision, thalamus

Edited by John Foxe

Received 7 June 2016, revised 5 July 2016, accepted 6 July 2016

\begin{abstract}
Neuroplasticity - the capacity of the brain to change as a response to internal and external pressures - has been studied from a number of different perspectives. Perhaps one of the most powerful models is the study of populations that have been congenitally deprived of a sense. It has been shown that the right Auditory Cortex (AC) of congenitally deaf humans is neuroplastically modified in order to represent visual properties of a stimulus. One unresolved question is how this visual information is routed to the AC of congenitally deaf individuals. Here, we performed volumetric analysis of subcortical auditory and visual brains regions namely the thalamus (along with three thalamic nuclei: the pulvinar, the lateral geniculate nucleus and the medial geniculate nucleus), and the inferior and superior colliculi - in deaf and hearing participants in order to identify which structures may be responsible for relaying visual information toward the altered AC. Because there is a hemispheric asymmetry in the neuroplastic changes observed in the AC of the congenitally deaf, we reasoned that subcortical structures that also showed a similar asymmetry in their total volume could have been enlisted in the effort of relaying visual information to the neuroplastically altered right AC. We show that for deaf, but not for hearing individuals, the right thalamus, right lateral geniculate nucleus and right inferior colliculus are larger than their left counterparts. These results suggest that these subcortical structures may be responsible for rerouting visual information to the $A C$ in congenital deafness.
\end{abstract}

\section{Introduction}

The remarkable capacity of the brain to neuroplastically change itself as a response to internal and external pressures is an important and established fact (e.g., Kaas, 1991; Bavelier \& Neville, 2002;

Correspondence: Jorge Almeida, ${ }^{1}$ Proaction Laboratory, as above.

E-mail: jorgealmeida@fpce.uc.pt
Amaral \& Almeida, 2015). The study of congenital sensorial deprivation, and in particular congenital deafness, has made decisive contributions to our understanding of how long-term neuroplasticity unfolds in the brain and how it impacts behavior (e.g. Finney et al., 2001; Emmorey et al., 2003; Kral et al., 2003; Fine et al., 2005; Bavelier et al., 2006; Hunt et al., 2006; Lomber et al., 2010; Meredith \& Lomber, 2011; Meredith et al., 2011; Lyness et al., 
2014; Scott et al., 2014; Almeida et al., 2015; Wang et al., 2015). For instance, it has been shown that the right auditory cortex (AC) of congenitally deaf humans responds to visual stimuli presented in the visual periphery (e.g. Finney et al., 2001, 2003; Almeida et al., 2015; but see Scott et al., 2014). One issue that remains unresolved is how information from the unaffected senses is rerouted toward the sensory cortices that typically process information from the affected sense. That is, what structures are enlisted in the putative novel route that takes visual information to the right AC of congenitally deaf humans? Here, we address this question by studying volumetric changes in subcortical auditory and visual relay structures in congenitally deaf subjects in whom we have demonstrated (Almeida et al., 2015) the hemispheric asymmetry, whereby their right $\mathrm{AC}$ processes visual information.

Research with congenitally deaf humans has shown that regions putatively associated with auditory processing - for example, the AC - are co-opted to process information from other senses. Finney et al. (2001; see also Levanen et al., 1998; Nishimura et al., 1999; Finney et al., 2003; Karns et al., 2012; Almeida et al., 2015; but see Hickok et al., 1997; Scott et al., 2014) measured neuronal activity in deaf and hearing individuals under simple visual stimulation, and found that for deaf, but not hearing individuals, the right $\mathrm{AC}$ was responsive to visual stimulation. Moreover, Almeida et al. (2015) showed that the right AC of congenitally deaf humans contains information about whether a stimulus is presented in the center of the visual field or in the periphery. Importantly, data from studies with congenitally deaf animals support the role of the AC in processing information from the unaffected senses - in particular, visual and somatosensory stimuli (e.g. Rebillard et al., 1977, 1980; Kral et al., 2003; Hunt et al., 2006; Meredith \& Lomber, 2011). Moreover, the AC shows selectivity to properties that are typically visual (e.g. direction selectivity; Meredith \& Lomber, 2011)

The fact that visual stimuli are processed in auditory regions in deaf individuals leaves open questions about how that information reaches the neuroplastically changed AC. What is the (potentially novel) route from the retina to the $\mathrm{AC}$ that provides right $\mathrm{AC}$ with visual information? What structures are enlisted in that effort? In an influential paper, Bavelier \& Neville (2002) proposed four types of mechanisms that could account for the neuroplastic changes happening in sensory cortices. According to those authors, rerouting of spared sensory information could be related to changes in local connectivity, changes in subcortical connectivity, changes in cortico-cortical feedback and/or changes in long-range cortico-cortical connectivity.

Extant data seem to suggest that subcortical relay structures have an important role in the rerouting of this information. For instance, in newborn ferrets in which retinal cells were redirected into the medial geniculate nucleus (i.e. the principal auditory thalamic nucleus), the response patterns to visual stimulation in primary AC were similar to the ones observed in primary visual cortex in that they showed selectivity to similar properties (e.g. direction and orientation selectivity; Sur et al., 1988; Roe et al., 1990). Moreover, Barone et al. (2013) showed that visual thalamic nuclei project to the AC of congenitally deaf cats. In accordance with these data, Lyness et al. (2014) have shown that congenital deafness imparted neuroplastic changes to the white matter thalamic-cortical tracts in humans. Finally, the work of Knudsen and colleagues (Knudsen et al., 1991, 1993; Knudsen \& Knudsen, 1996; Knudsen, 2002) on multimodal mapping of space in the inferior colliculi of the barn owl suggests that those subcortical structures may be capable of passing on and representing different types of sensorial information. These data support the hypothesis that visual information can be passed on to the AC through subcortical structures.
To test this hypothesis we will focus on a series of candidate subcortical structures that are the major relay structures of visual and auditory information to the cortex. In particular we focus on: (i) the Thalamus (Thal), which is the main relay site of sensorial (auditory and visual) information toward the cortex and is a major component of the diencephalon; (ii) the Pulvinar (Pul), which is the largest nuclei of the Thal, and is involved in the processing of visual information coming both from cortical associate areas, as well as other subcortical structures such as the superior colliculi (e.g. Smith et al., 2009); (iii) the Lateral Geniculate Nucleus (LGN), which is the visual thalamic relay structure that passes visual information from the retina to the primary visual cortex and has been shown to have direct connections with the AC of deaf cats (e.g., Barone et al., 2013); (iv) the Medial Geniculate Nucleus (MGN), which is the auditory thalamic relay, is an integral part of the ascending auditory pathway toward the AC in hearing individuals (e.g. Butler \& Lomber, 2013), and seems to maintain its connectivity with the AC under congenital deafness (Stanton \& Harrison, 2000); (v) the Superior Colliculus (SC), which is a subcortical structure located in the midbrain that receives direct retinal input (e.g. Schiller \& Malpeli, 1977), and contains an orderly representation of the visual field (e.g. Cynader \& Berman, 1972); and (vi) the Inferior Colliculus (IC), which is a subcortical structure, inferior to the SC, that is an integral part of the ascending auditory pathway that passes auditory information from subcortical auditory structures upstream, such as the cochlear nuclei, superior olives and nuclei of the lateral lemniscus, to the AC via auditory thalamus (e.g. Butler \& Lomber, 2013).

We use MRI to measure volumetric changes in these auditory and visual subcortical relay structures in congenitally deaf individuals, compared to a reference group of hearing individuals. We take advantage of the hemispheric asymmetry of the neuroplastic changes present in the AC of congenitally deaf humans (e.g. Finney et al., 2001, 2003; Almeida et al., 2015; but see Scott et al., 2014). Specifically, we reason that subcortical structures that are the major relay of visual and auditory information to the cortex and that also show a similar asymmetry in their total volume - larger volumes in the right than in the left hemisphere - are likely to have been enlisted in relaying visual information to the neuroplastically changed right $\mathrm{AC}$.

\section{Method}

\section{Participants}

Fifteen congenitally deaf individuals (13 women and 2 men, average age 20.4 years, range $17-22$ ) and 16 hearing individuals (13 women and 3 men, average age 20.1 years, range 18-22) participated in the experiment. All participants had normal or corrected-to-normal vision, had no history of neurological disorder, were right-handed and gave written informed consent in accordance with the institutional review board of Beijing Normal University (BNU) Imaging Center for Brain Research. All congenitally deaf participants were native signers of Chinese sign language, had hearing loss above $90 \mathrm{~dB}$ binaurally (frequencies tested ranged from 125 to $8000 \mathrm{~Hz}$ ) and did not benefit from the use of hearing aids (used, in the past, by five of the participants). All hearing participants reported no hearing impairment or knowledge of Chinese sign language. A large subset of these participants (10 hearing and 10 deaf) also participated in the experiments reported in Almeida et al. (2015); see also Wang et al., 2015). The study was approved by the institutional review board of the Beijing Normal University (BNU) Imaging 
Center for Brain Research. The study conforms to World Medical Association Declaration of Helsinki.

\section{MRI Structural image acquisition}

The MRI data were collected at the BNU MRI center on a $3 \mathrm{~T}$ Siemens Trio Tim scanner. A high-resolution 3D structural data set was acquired with a 3D-MPRAGE sequence in the sagittal plane (TR: $2530 \mathrm{~ms}$, TE: $3.39 \mathrm{~ms}$, flip angle: 7 degrees, matrix size: $256 \times 256$, voxel size: $1.33 \times 1 \times 1.33 \mathrm{~mm}, 144$ slices, acquisition time: $8.07 \mathrm{~min})$.

\section{Preprocessing}

The structural imaging data were reconstructed and corrected using FreeSurfer 5.0 (Fjell et al., 2008; Hodneland et al., 2012; Reuter et al., 2012). The processing steps consisted of motion and intensity correction, normalization to the Tailarach template (Talairach \& Tournoux, 1988), skull stripping and segmentation (Fischl, 2012). The data reconstruction steps were manually checked for accuracy.

\section{Manual segmentation}

Regions-of-interest (ROIs) were traced by hand on contiguous slices of the preprocessed high-resolution T1-weighted images of the brain using 3D Slicer v.2.7 (http://www.slicer.org; Fedorov et al., 2012). As described above, our target ROIs included the Thalamus (Thal), the Pulvinar (Pul), the Lateral Geniculate Nucleus (LGN), the Medial Geniculate Nucleus (MGN), the Superior Colliculi (SC) and the Inferior Colliculi (IC). Delineation of these ROIs, in each hemisphere, followed protocols already implemented in the literature (Fig. 1). In particular, to define the borders of the Thal we followed Portas et al. (1998). The Thal is anteriorly defined by the mamillary bodies of the hypothalamus, with the posterior border limited to the point where the Thal merges under the crus fornix. Medially, the Thal is defined by the third ventricle, and its inferior and superior boundaries are defined by the brain stem and the main body of the lateral ventricle respectively. We used the protocol proposed by Byne et al. (2001) to delineate the borders of the Pul. The Pul is posteromedially defined by the cerebrospinal fluid and anteromedially by the posterior border of the mediodorsal nucleus. The lateral border is established by the internal capsule and the anterior border is defined laterally by the corticotectal tract. The inferior border, although difficult to define, usually corresponds to the limit or slightly above the level of the SC. The LGN is located above the hippocampus and the ambient cistern and laterally below the Thalamus. Laterally, the LGN is limited by the optic radiations (Korsholm et al., 2007). The MGN was defined according to the atlas in Tamraz \& Comair (2006). The MGN is defined superiorly by the peduncle. Laterally, it is located below the Pulvinar and the Thalamus. The atlas proposed by Tamraz \& Comair (2006) was also used to define the borders of both SC and IC. The SC is defined superiorly by the posterior commissure (PC) and inferiorly by the IC. Anteriorly, it is delimited by the commissure of SC exclusive, which is the white tract visualized immediately below PC. The IC is defined superiorly by the SC and inferiorly by the superior medullary velum. Laterally, the IC is limited by the ambient cistern (Fig. 1).

\section{Reliability}

For all participants, the ROIs were manually traced by one of the authors (L.A.). Segmentation reliability was assessed by having two
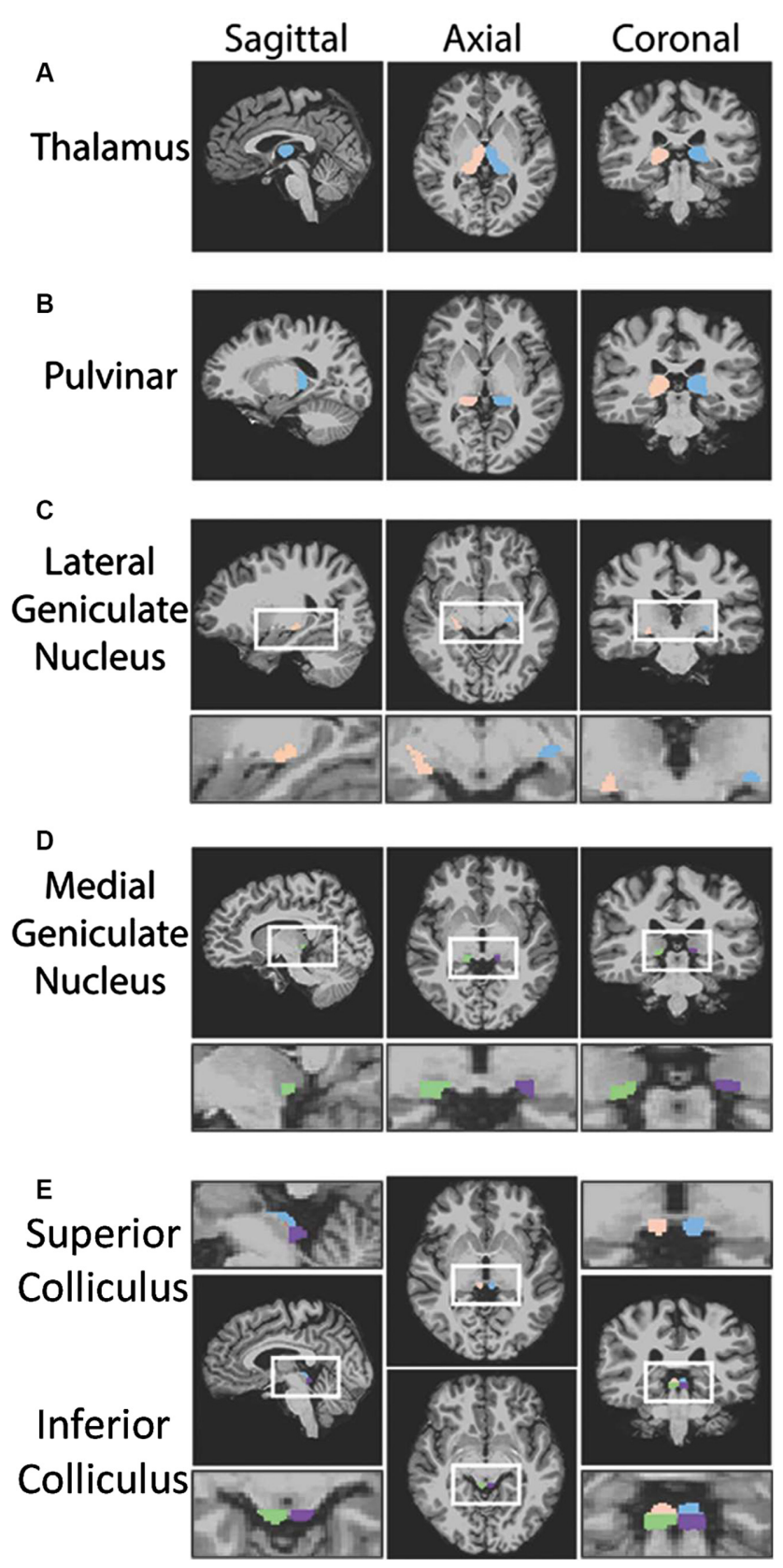

FIG. 1. Examples of the manually segmented ROIs. Examples of the segmented ROIs in axial, coronal and sagittal images in one participant. (A) Thal; (B) Pul; (C) LGN; (D) MGN; (E) SC and IC. The Talairach coordinates of the different planes for each ROI are: for the Thal - sagittal $\mathrm{x}=-4$, coronal $\mathrm{y}=-20$ and axial $\mathrm{z}=2$; for the Pul - sagittal $\mathrm{x}=-22$; coronal $\mathrm{y}=-18$ and axial $\mathrm{z}=2$; for the $\mathrm{LGN}-$ sagittal $\mathrm{x}=21$, coronal $\mathrm{y}=-12$ and axial $\mathrm{z}=-7$; for the MGN - sagittal $\mathrm{x}=9$, coronal $\mathrm{y}=-19$ and axial $\mathrm{z}=-3$; for the IC - sagittal $\mathrm{x}=-6$, coronal $\mathrm{y}=-22$ and axial $\mathrm{z}=-8$; and for the $\mathrm{SC}-$ sagittal $\mathrm{x}=-6$, coronal $\mathrm{y}=-22$ and axial $\mathrm{z}=-2$. ROIs defined in blue and purple are within the left hemisphere, whereas those defined in orange and green are in the right hemisphere.

other authors (A.G-A.; M.J.S.) trace the ROIs in four of the brains (eight hemispheres) randomly selected from the pool of participants in the study. In order to keep the investigators naive, access to the status of each participant was known only after the segmentation of all ROIs. We calculated intraclass coefficients for each of the ROIs in the four brains that were traced by both authors. The obtained 
Cronbach's Alphas show high-reliability coefficients for every ROI (left Thal: $\alpha=0.95$; right Thal: $\alpha=0.99$; left Pul: $\alpha=0.99$; right Pul: $\alpha=0.98$; left LGN: $\alpha=0.98$; right LGN: $\alpha=0.98$; left MGN: $\alpha=0.96$; right MGN: $\alpha=0.95$; left SC $\alpha=0.80$; right SC $\alpha=0.94$; left IC $\alpha=0.86$; right IC $\alpha=0.83$; all values at $P<0.05)$.

\section{Data analysis}

We first computed a 6 (ROI: Thal, Pul, LGN, MGN, SC and IC) X 2(Hemisphere: right vs. left) X 2(Group: deaf vs. hearing) ANOvA, with the first two factors as within-participants factors, and the remaining factor as a between-participants factor. From this ANOVA, we inspected the three-way interaction, and then used planned contrasts to analyze the question of interest - are there hemispheric asymmetries in the total volume for each ROI (Thal, Pul, SC, and IC) by group (Deaf and Hearing).

\section{Results}

Table 1 presents the average total volume for each target ROI by group membership for each hemisphere. Our three-way interaction between ROI, hemisphere and group membership was significant $\left(F_{1.5,43.8}=5.42 ; \quad P=0.014 ;\right.$ Greenhouse-Geisser corrected $]$. We then focused on our questions of interest and further unpacked the three-way interaction by calculating contrasts to test whether there were hemispheric asymmetries in each ROI for each group independently. Planned contrasts performed over the data for the hearing group failed to reveal any significant hemispheric asymmetry [all $t<1$; except for the LGN: $t_{15}=1.89 ; P=0.078$ ]. Importantly, the deaf group presented hemispheric asymmetries for some of the target ROIs in the hypothesized direction. Specifically, the right Thal was significantly larger than the left Thal $\left(t_{14}=4.287\right.$; $P=0.00075)$. This was the only ROI that showed a hemispheric asymmetry that survives a Bonferroni correction for multiple comparisons. However, if we use more lenient criteria (i.e. non-corrected comparisons), the right IC also showed larger total volume than the left IC $\left(t_{14}=2.477 ; P=0.027\right)$. Finally, the right LGN was marginally larger than the left LGN $\left(t_{14}=2.025 ; P=0.062\right)$. The remaining comparisons for the deaf group did not show any hemispheric asymmetries (for the Pul: $t_{14}=1.115 ; P=0.284$; for the MGN: $t<1$; for the SC: $t_{14}=1.294 ; P=0.217$ ). For comparisons between the volumetric ratios of deaf and hearing participants please see Table S1.

TABLE 1 . Volumes $\left(\mathrm{cm}^{3}\right)$ of ROIs and asymmetry statistics

\begin{tabular}{lllll}
\hline & $\begin{array}{l}\text { Status } \\
\text { ROI }\end{array}$ & $\begin{array}{l}\text { Left volume } \\
\text { (Hearing; } n=16)\end{array}$ & $\begin{array}{l}\text { Right volume } \\
\text { Mean (SD) }\end{array}$ & Pairwise $t$-test \\
\hline \multirow{2}{*}{ Thal } & Deaf & $5.676(0.735)$ & $6.078(0.958)$ & $t_{14}=4.287 * *$ \\
& Hearing & $5.830(0.989)$ & $5.806(0.996)$ & $t_{15}=0.278$ \\
Pul & Deaf & $1.235(0.313)$ & $1.358(0.332)$ & $t_{14}=1.115$ \\
& Hearing & $1.498(0.323)$ & $1.458(0.396)$ & $t_{15}=0.414$ \\
LGN & Deaf & $0.214(0.055)$ & $0.235(0.045)$ & $t_{14}=2.025^{¥}$ \\
& Hearing & $0.274(0.092)$ & $0.250(0.089)$ & $t_{15}=1.890$ \\
MGN & Deaf & $0.111(0.036)$ & $0.109(0.027)$ & $t_{14}=0.160$ \\
& Hearing & $0.115(0.019)$ & $0.116(0.034)$ & $t_{15}=0.186$ \\
& Deaf & $0.117(0.035)$ & $0.128(0.035)$ & $t_{14}=1.294$ \\
IC & Hearing & $0.176(0.038)$ & $0.171(0.034)$ & $t_{15}=0.525$ \\
& Deaf & $0.168(0.025)$ & $0.185(0.036)$ & $t_{14}=2.477 *$ \\
& Hearing & $0.193(0.034)$ & $0.194(0.040)$ & $t_{15}=0.182$ \\
\hline \multirow{2}{*}{ SC } & & & &
\end{tabular}

${ }^{¥} P=0.06 ; * P<0.05 ; * * P<0.0001$.

\section{Discussion}

It is an established fact that the brain is capable of neuroplastically changing itself as a response to internal and external pressures. Congenital deafness has been an important model for understanding how long-term neuroplasticity is implemented in the brain. For instance, the auditory cortex (AC) of congenitally deaf individuals can be co-opted to process visual (and somatosensory) stimuli (e.g. Rebillard et al., 1977, 1980; Levanen et al., 1998; Nishimura et al., 1999; Finney et al., 2001, 2003; Kral et al., 2003; Hunt et al., 2006; Meredith \& Lomber, 2011; Karns et al., 2012; Scott et al., 2014; Almeida et al., 2015), and has been shown to represent properties typical of the visual cortex (e.g. Almeida et al., 2015). An unsolved issue concerns how visual information reaches the $\mathrm{AC}$ in congenitally deaf individuals. To address this issue, we focused on subcortical structures that typically relay visual and auditory information to the cortex and measured their volumetric properties. We found that in deaf, but not in hearing human participants, the Thal (including the LGN, but excluding the Pul and the MGN), and the IC present hemispheric asymmetries that are similar to those observed for the functional processing of visual information in the AC.

Processing and representation of visual information in the AC of congenitally deaf humans is biased toward the right hemisphere (e.g. Finney et al., 2001, 2003; Almeida et al., 2015; but see Scott et al., 2014). This hemispheric asymmetry in the processing of visual information under congenital deafness toward the right $\mathrm{AC}$ should leave a signature upstream in the structures involved in passing visual information from the retina to the neuroplastically transformed AC. That is, those regions that are enlisted in the passing of visual information to the reorganized AC should also show hemispheric asymmetries of the kind observed for the functional processing of visual information under deafness. Moreover, this asymmetry should be visible on their total volume - all else being equal, these regions would have gained extra connections and functions if they were to be involved in the (novel) route that would lead visual information from the retina to the modified AC. This is exactly what our data demonstrate - the right Thal, right LGN and right IC show larger volumes than the left counterparts, and this difference is present in deaf and not hearing individuals suggesting it is a result of the neuroplastic forces at play. Importantly, a large subset of our participants took part in the study of Almeida et al. (2015), and hence present the hemispheric asymmetry toward the right $\mathrm{AC}$ for the processing of peripheral vs. centrally presented stimuli.

Our results are in accordance with extant data on the role of these subcortical structures in passing visual information toward the $\mathrm{AC}$ in individuals with congenital deafness. Specifically, both visual and auditory thalamic relays have been shown to undergo neuroplastic changes in congenital deafness (Lyness et al., 2014), and to be capable of relaying visual information to the AC (Sur et al., 1988; Roe et al., 1990; Barone et al., 2013). Moreover, the IC has been consistently shown to be well placed for integrating sensory information from multiple senses (e.g. Brainard \& Knudsen, 1993; Hyde \& Knudsen, 2001; Knudsen, 2002). For instance, in the barn owl, this structure integrates auditory and visual information when computing spatial maps of the surrounding environment. This multimodal nature of the IC may turn out to be optimal for relaying visual information to a cortical location that is typically dedicated to auditory processing (i.e. the $\mathrm{AC}$ ) or to other relay structures such as the LGN.

Our data are silent, however, in regard to the functional role of these structures in relaying visual information to the neuroplastically 
changed AC, as well as their impact on the processing of visual information in congenitally deaf individuals. The Thal is a subcortical region heavily involved in relaying visual and auditory information to primary sensory cortical areas. It is constituted by different nuclei, including the Pul, the LGN, and the MGN. According to our data, the Pul and the MGN do not show the asymmetric bias and as such may not be centrally involved in the passing of visual information to the $\mathrm{AC}$ of deaf individuals.

Our data are, however, suggestive of an important role of the LGN (and potentially other thalamic nuclei) in relaying visual information to the modified AC. Beyond the hemispheric asymmetry presented here, there are other processing characteristics that may implicate the LGN in the passing of visual information to the reorganized AC. Almeida et al. (2015) showed that the crude representation of the visual field within the changed AC under deafness is skewed - there are graded responses to locations within the horizontal plane, but not so much within the vertical plane. Importantly, the LGN also shows a somewhat skewed representation of the visual field, showing a similar preference for the horizontal plane (e.g. Schneider et al., 2004). As such, some of the processing characteristics of the AC in the deaf may be related with the kind of processing happening within the LGN.

One interesting aspect of our data concerns the fact that deaf participants present volumetric reductions (at least numerically), across the board, for the subcortical structures analyzed. A possible explanation for this rests on the fact that the usual connections toward the AC are rendered unnecessary and are pruned or degenerate, leading to volumetric reduction. Perhaps the rate of neuroplastically induced growth in these regions in order to relay visual information to the $\mathrm{AC}$ is not sufficient to counteract processes of pruning or degeneration to maintaining the typical sizes of these structures.

It is important to note also that not all reports on the processing of visual stimuli within the AC show strong laterality toward the right hemisphere. For instance, Scott et al. (2014) showed that the left and right $\mathrm{AC}$ respond to visual stimuli. However, these authors restricted their visual presentation to the right visual field. Interestingly, then, visual responses in the right AC are elicited by ipsilateral presentations - that is, even in situations where visual information is presented to the ipsilateral visual field, the right $\mathrm{AC}$ is robustly activated.

Finally, an important implication of these results concerns the success rate of cochlear implants, and generally any sensory substitution device, on congenital sensorial deprivation. By showing that cortical structures upstream are already subject to important neuroplastic changes, our data may suggest that the processing characteristics of those subcortical structures may also have to be taken into consideration alongside the $\mathrm{AC}$ and the cochlea when designing these devices.

\section{Supporting Information}

Additional supporting information can be found in the online version of this article:

Table S1. Volumes $\left(\mathrm{cm}^{3}\right)$ of ROIs and statistical comparisons of deaf and hearing participants.

\section{Acknowledgements}

LA is supported by Fundação BIAL (http://www.bial.com/en/). AG-A is supported by Fundação para a Ciência e a Tecnologia Doctoral Grant number SFRH/BD/80945/2011 and Programa COMPETE. AO is funded by São
Paulo Research Foundation (FAPESP) grant number 2014/06777-0, by the National Council for Scientific and Technological Development (CNPq) grant number 487188/2013-6 and by MackPesquisa grant number $105 / 2015$. FF is funded by the NSFC of China (30925014; 31230029). DH is supported in part by a Postdoctoral Fellowship funded by the Peking-Tsinghua Center for Life Sciences. BZM and QC were supported by NIH grant R01NS089609 to BZM. YB is funded by NNSF of China (31171073; 31222024). JA is funded by Fundação para a Ciência e a Tecnologia projects PTDC/MHC-PCN/6805/2014 and PTDC/MHC-PCN/0522/2014, Programa COMPETE and by Fundação BIAL 112/12. This research was supported by Fundação BIAL (http://www.bial.com/en/).

\section{Abbreviations}

AC, Auditory Cortex; IC, Inferior Colliculus; LGN, Lateral Geniculate Nucleus; MGN, Medial Geniculate Nucleus; Pul, Pulvinar; SC, Superior Colliculus; Thal, Thalamus.

\section{References}

Almeida, J., He, D., Chen, Q., Mahon, B.Z., Zhang, F., Goncalves, O.F., Fang, F. \& Bi, Y. (2015) Decoding visual location from neural patterns in the auditory cortex of the congenitally deaf. Psychol. Sci., 26, 1771-1782.

Amaral, L. \& Almeida, J. (2015) Neuroplasticity in congenital deaf humans. Rev. Port. Psicol., 44, 39-45.

Barone, P., Lacassagne, L. \& Kral, A. (2013) Reorganization of the connectivity of cortical field DZ in congenitally deaf cat. PLOS ONE, 8, e60093.

Bavelier, D. \& Neville, H.J. (2002) Cross-modal plasticity: where and how? Nat. Rev. Neurosci., 3, 443-452.

Bavelier, D., Dye, M.W. \& Hauser, P.C. (2006) Do deaf individuals see better? Trends Cogn. Sci., 10, 512-518.

Brainard, M.S. \& Knudsen, E.I. (1993) Experience-dependent plasticity in the inferior colliculus: a site for visual calibration of the neural representation of auditory space in the barn owl. J. Neurosci., 13, 4589-4608.

Butler, B.E. \& Lomber, S.G. (2013) Functional and structural changes throughout the auditory system following congenital and early-onset deafness: implications for hearing restoration. Front. Syst. Neurosci., 7, 92.

Byne, W., Buchsbaum, M.S., Kemether, E., Hazlett, E.A., Shinwari, A., Mitropoulou, V. \& Siever, L.J. (2001) Magnetic resonance imaging of the thalamic mediodorsal nucleus and pulvinar in schizophrenia and schizotypal personality disorder. Arch. Gen. Psychiat., 58, 133-140.

Cynader, M. \& Berman, N. (1972) Receptive-field organization of monkey superior colliculus. J. Neurophysiol., 35, 187-201.

Emmorey, K., Allen, J.S., Bruss, J., Schenker, N. \& Damasio, H. (2003) A morphometric analysis of auditory brain regions in congenitally deaf adults. Proc. Natl. Acad. Sci. USA, 100, 10049-10054.

Fedorov, A., Beichel, R., Kalpathy-Cramer, J., Finet, J., Fillion-Robin, J.-C., Pujol, S., Bauer, C., Jennings, D. et al. (2012) 3D Slicer as an image computing platform for the Quantitative Imaging Network. Magn. Reson. Imaging, 30, 1323-1341.

Fine, I., Finney, E.M., Boynton, G.M. \& Dobkins, K.R. (2005) Comparing the effects of auditory deprivation and sign language within the auditory and visual cortex. J. Cogn. Neurosci., 17, 1621-1637.

Finney, E.M., Fine, I. \& Dobkins, K.R. (2001) Visual stimuli activate auditory cortex in the deaf. Nat. Neurosci., 4, 1171-1173.

Finney, E.M., Clementz, B.A., Hickok, G. \& Dobkins, K.R. (2003) Visual stimuli activate auditory cortex in deaf subjects: evidence from MEG. NeuroReport, 14, 1425-1427.

Fischl, B. (2012) FreeSurfer. Neurolmage, 62, 774-781.

Fjell, A.M., Westlye, L.T., Greve, D.N., Fischl, B., Benner, T., van der Kouwe, A.J., Salat, D., Bjornerud, A. et al. (2008) The relationship between diffusion tensor imaging and volumetry as measures of white matter properties. NeuroImage, 42, 1654-1668.

Hickok, G., Poeppel, D., Clark, K., Buxton, R.B., Rowley, H.A. \& Roberts, T.P. (1997) Sensory mapping in a congenitally deaf subject: MEG and fRMI studies of cross-modal non-plasticity. Hum. Brain Mapp., 5, 437-444.

Hodneland, E., Ystad, M., Haasz, J., Munthe-Kaas, A. \& Lundervold, A. (2012) Automated approaches for analysis of multimodal MRI acquisitions in a study of cognitive aging. Comp. Meth. Prog. Bio., 106, $328-341$.

Hunt, D.L., Yamoah, E.N. \& Krubitzer, L. (2006) Multisensory plasticity in congenitally deaf mice: how are cortical areas functionally specified? Neuroscience, 139, 1507-1524. 
Hyde, P.S. \& Knudsen, E.I. (2001) A topographic instructive signal guides the adjustment of the auditory space map in the optic tectum. J. Neurosci., 21, 8586-8593.

Kaas, J.H. (1991) Plasticity of sensory and motor maps in adult mammals. Annu. Rev. Neurosci., 14, 137-167.

Karns, C.M., Dow, M.W. \& Neville, H.J. (2012) Altered cross-modal processing in the primary auditory cortex of congenitally deaf adults: a visual-somatosensory fMRI study with a double-flash illusion. J. Neurosci., 32, 9626-9638.

Knudsen, E.I. (2002) Instructed learning in the auditory localization pathway of the barn owl. Nature, 417, 322-328.

Knudsen, E.I. \& Knudsen, P.F. (1996) Disruption of auditory spatial working memory by inactivation of the forebrain archistriatum in barn owls. Nature, 383, 428-431.

Knudsen, E.I., Esterly, S.D. \& du Lac, S. (1991) Stretched and upside-down maps of auditory space in the optic tectum of blind-reared owls; acoustic basis and behavioral correlates. J. Neurosci., 11, 1727-1747.

Knudsen, E.I., Knudsen, P.F. \& Masino, T. (1993) Parallel pathways mediating both sound localization and gaze control in the forebrain and midbrain of the barn owl. J. Neurosci., 13, 2837-2852.

Korsholm, K., Madsen, K.H., Frederiksen, J.L., Skimminge, A. \& Lund, T.E. (2007) Recovery from optic neuritis: an ROI-based analysis of LGN and visual cortical areas. Brain, 130, 1244-1253.

Kral, A., Schroder, J.H., Klinke, R. \& Engel, A.K. (2003) Absence of crossmodal reorganization in the primary auditory cortex of congenitally deaf cats. Exp. Brain Res., 153, 605-613.

Levanen, S., Jousmaki, V. \& Hari, R. (1998) Vibration-induced auditory-cortex activation in a congenitally deaf adult. Curr. Biol., 8, 869-872.

Lomber, S.G., Meredith, M.A. \& Kral, A. (2010) Cross-modal plasticity in specific auditory cortices underlies visual compensations in the deaf. Nat. Neurosci., 13, 1421-1427.

Lyness, R.C., Alvarez, I., Sereno, M.I. \& MacSweeney, M. (2014) Microstructural differences in the thalamus and thalamic radiations in the congenitally deaf. NeuroImage, 100, 347-357.

Meredith, M.A. \& Lomber, S.G. (2011) Somatosensory and visual crossmodal plasticity in the anterior auditory field of early-deaf cats. Hear. Res., 280, 38-47.

Meredith, M.A., Kryklywy, J., McMillan, A.J., Malhotra, S., Lum-Tai, R. \& Lomber, S.G. (2011) Crossmodal reorganization in the early deaf switches sensory, but not behavioral roles of auditory cortex. Proc. Natl. Acad. Sci. USA, 108, 8856-8861.

Nishimura, H., Hashikawa, K., Doi, K., Iwaki, T., Watanabe, Y., Kusuoka, H., Nishimura, T. \& Kubo, T. (1999) Sign language 'heard' in the auditory cortex. Nature, 397, 116.
Portas, C.M., Goldstein, J.M., Shenton, M.E., Hokama, H.H., Wible, C.G., Fischer, I., Kikinis, R., Donnino, R. et al. (1998) Volumetric evaluation of the thalamus in schizophrenic male patients using magnetic resonance imaging. Biol. Psychiat., 43, 649-659.

Rebillard, G., Carlier, E., Rebillard, M. \& Pujol, R. (1977) Enhancement of visual responses on the primary auditory cortex of the cat after an early destruction of cochlear receptors. Brain Res., 129, 162 164.

Rebillard, G., Rebillard, M. \& Pujol, R. (1980) Factors affecting the recording of visual-evoked potentials from the deaf cat primary auditory cortex (AI). Brain Res., 188, 252-254.

Reuter, M., Schmansky, N.J., Rosas, H.D. \& Fischl, B. (2012) Within-subject template estimation for unbiased longitudinal image analysis. NeuroImage, 61, 1402-1418.

Roe, A.W., Pallas, S.L., Hahm, J.O. \& Sur, M. (1990) A map of visual space induced in primary auditory cortex. Science, $\mathbf{2 5 0}, 818-820$.

Schiller, P.H. \& Malpeli, J.G. (1977) Properties and tectal projections of monkey retinal ganglion cells. J. Neurophysiol., 40, 428-445.

Schneider, K.A., Richter, M.C. \& Kastner, S. (2004) Retinotopic organization and functional subdivisions of the human lateral geniculate nucleus: a high-resolution functional magnetic resonance imaging study. J. Neurosci., 24, 8975-8985.

Scott, G.D., Karns, C.M., Dow, M.W., Stevens, C. \& Neville, H.J. (2014) Enhanced peripheral visual processing in congenitally deaf humans is supported by multiple brain regions, including primary auditory cortex. Front. Hum. Neurosci., 8, 177.

Smith, A.T., Cotton, P.L., Bruno, A. \& Moutsiana, C. (2009) Dissociating vision and visual attention in the human pulvinar. J. Neurophysiol., 101, 917-925.

Stanton, S.G. \& Harrison, R.V. (2000) Projections from the medial geniculate body to primary auditory cortex in neonatally deafened cats. J. Comp. Neurol., 426, 117-129.

Sur, M., Garraghty, P.E. \& Roe, A.W. (1988) Experimentally induced visual projections into auditory thalamus and cortex. Science, 242, 14371441.

Talairach, J. \& Tournoux, P. (1988) Co-Planar Stereotaxic Atlas of the Human Brain. 3-Dimensional Proportional System: An Approach to Cerebral Imaging. Thieme, New York.

Tamraz, J.C. \& Comair, Y.G. (2006) Atlas of Regional Anatomy of the Brain Using MRI. Springer, Berlin.

Wang, X., Caramazza, A., Peelen, M.V., Han, Z. \& Bi, Y. (2015) Reading without speech sounds: VWFA and its connectivity in the congenitally deaf. Cereb. Cortex, 25, 2416-2426. 